\title{
Bounds on the Unstable Eigenvalue for Period Doubling
}

\author{
J.-P. Eckmann ${ }^{1}$ and H. Epstein ${ }^{2}$ \\ ${ }^{1}$ Département de Physique Théorique, Université de Genève, CH-1211 Genève 4, Switzerland \\ ${ }^{2}$ CNRS and IHES, Bures sur Yvette, France
}

\begin{abstract}
Bounds are given for the unstable eigenvalue of the period-doubling operator for unimodal maps of the interval. These bounds hold for all types of behaviour $|x|^{r}$ of the interval map near its critical point. They are obtained by finding cones in function space which are invariant under the tangent map to the doubling operator at its fixed point.
\end{abstract}

\section{Introduction}

One-parameter families of maps of the interval, such as

$$
\mu \mapsto 1-\mu|x|^{r},
$$

with $r>1$ fixed, exhibit sequences of bifurcation points for period doubling, which accumulate at a universal rate $\delta_{r},[F, C T]$. For example, for families of quadratic maps (i.e., for $r=2$ ), it is well known that $\delta_{r}=4.66920 \ldots$. A rigorous bound on $\delta_{2}$ is given in [EW 2]. The aim of this paper is to give rigorous bounds on $\delta_{r}$ for all $r>1$ by using a convenient reformulation of the tangent map to the doubling operator.

To state these bounds, we need some notation. Consider the CvitanovićFeigenbaum equation

$$
g(x)=-\frac{1}{\lambda} g(g(-\lambda x)),
$$

with the conditions $g(0)=1$ and $\lambda=-g(1)$. We consider this equation on several classes of functions, labelled by $r$. Namely, we require $g$ to be of the form $g(x)$ $=f\left(|x|^{r}\right)$ with $f$ analytic in a neighborhood of $[0,1]$. A solution of this equation is then denoted by $g_{r}$. Solutions having some additional properties (we shall spell them out in the next section) are known to exist for all $r>1$. In this paper, we show that these additional properties have the following consequence: Denote $f_{r}(z)$ $=g_{r}\left(z^{r}\right)$ for $z \in[0,1]$, and $\lambda_{r}=-g_{r}(1)$. Then, $\delta_{r}$ satisfies the inequality

$$
\frac{1}{\lambda_{r}^{r}}\left(1-\frac{1}{r\left|f_{r}^{\prime}(0)\right|}\right)<\delta_{r}<\frac{1}{\lambda_{r}^{r}} .
$$


To prove the above statement, we show that the tangent map of the doubling operator preserves a certain cone in function space (improving thus on a previous similar result of [CE] valid only for $r \leqq 2$ ). Below, we begin by fixing the notation and performing changes of variables in function space. Then we develop the subject proper of this paper. Throughout the paper, $r$ is a fixed constant, $r>1$, and we drop henceforth the index $r$.

\section{General Notations}

We denote by $g$ a fixed point of the doubling operator, with the following properties:

1. $g$ is a continuous, strictly decreasing map of $[0,1]$ into $(-1,1]$, satisfying the Cvitanović-Feigenbaum equation:

$$
g(x)=-\frac{1}{\lambda} g(g(\lambda x)) \forall x \in[0,1], g(0)=1 .
$$

Here, $\lambda=-g(1) \in(0,1)$.

2. There is a function $f$, holomorphic on a complex neighborhood of $[0,1]$ such that

$$
g(x)=f\left(x^{r}\right), f^{\prime}(x)<0 \quad \forall x \in[0,1] .
$$

3. The inverse functions of $f$ and $g$ are respectively denoted $U$ and $u$. The function $U$ extends to a function which is holomorphic in the domain

$$
\Omega(\lambda)=\mathbf{C}_{+} \cup \mathbf{C}_{-} \cup\left(-\lambda^{-1}, \lambda^{-2}\right),
$$

where

$$
\mathbf{C}_{+}=-\mathbf{C}_{-}=\{z \in \mathbf{C}: \operatorname{Im} z>0\} \text {. }
$$

The function $U$ satisfies

$$
U\left(\mathbf{C}_{+}\right) \subset \mathbf{C}_{-}, \quad U\left(\mathbf{C}_{-}\right) \subset \mathbf{C}_{+} .
$$

Remark. It is extremely likely that all solutions of (2.1) with the properties 1) and 2) also have the property 3 ), since solutions having all three properties have been proved to exist for all $r>1$ (see [E3] and [E2] where a list of proofs of existence is given) and since, on the other hand, it is extremely likely that there is only one solution (for each $r$ ) having the properties 1) and 2). The validity of 3) can also be inferred from the "hyperbolicity" of $g$ if it holds. Finally it is in the process of being proved in a more general setting by D. Sullivan. In any case, we restrict henceforth our attention to those $g$ having the properties 1), 2), and 3). All other facts used in the sequel are proved consequences of these properties.

\section{Simple Consequences}

a) The equation $g(\lambda x)=x$ has a unique solution $x_{0}$ in $[0,1]$. It satisfies

$$
g\left(x_{0}\right)=0, g^{\prime}\left(\lambda x_{0}\right)=-1, \quad \lambda<x_{0}<1 .
$$

The inequality $\lambda<x_{0}$ holds since otherwise $g\left(x_{0} / \lambda\right)=-1 / \lambda$ would be in $[-1,1]$. 
b) The function $U$ is injective and bounded in $\Omega(\lambda)$, vanishes at 1, and has continuous boundary values [E2]. We denote $\Delta=U(\Omega(\lambda))$.

c) The function $u$ is anti-Herglotzian ${ }^{1}$ and holomorphic in $\mathbf{C}_{+} \cup \mathbf{C}_{-}$ $U\left(-\lambda^{-1}, 1\right)$, where it satisfies $u(z)=U(z)^{1 / r}$. For all $z \in \Omega(\lambda)$,

$$
U(z)=\frac{1}{\lambda^{r}} U(u(-\lambda z)) \text {. }
$$

As $r \rightarrow \infty$, the constants $\lambda$ and $x_{0}$ tend to 1 , and $U / U(0)$ tends to 1 uniformly on every compact in $\mathbf{C}_{+} \cup \mathbf{C}_{-} \cup(-1,1)$ [E1]. However, as shown in [EW], some objects have a non-trivial limiting behavior. Among them are the functions $L_{ \pm}$ defined below and the two quantities $\tau$ and $y_{0}$ which are given, for fixed but arbitrary $r$, by

$$
\tau=\lambda^{r}, y_{0}=x_{0}^{r} .
$$

Note that $y_{0}>\tau$. There are crude but uniform bounds on $\tau$ and $y_{0}$, e.g. $3<1 / \tau$ $<144 / \lambda, e^{-\lambda}<y_{0}<1 /(1+\lambda)([\mathrm{E} 1])$.

Definition. We denote by $q$ the map from $\mathbf{R}$ to $\mathbf{R}$ given by:

$$
q(x)=\operatorname{sign}(x)|x|^{r} \text {. }
$$

We then define $L$ by:

$$
L(z)=q(f(z)) \forall z \in[0,1] \text {, i.e. } L=q \circ g \circ q^{-1} .
$$

Note that $L$ has a zero of order $r$ at $y_{0}$. We shall also use:

$$
L_{+}(z)=L(z) \quad \text { on } \quad\left[0, y_{0}\right], \quad L_{-}(z)=-L(z) \text { on }\left[y_{0}, 1\right] .
$$

These functions satisfy the identities

$$
L(z)=-\frac{1}{\tau} L(L(\tau z)) \forall z \in[0,1],
$$

or, equivalently,

$$
\begin{aligned}
& L_{+}(z)=\frac{1}{\tau} L_{-}\left(L_{+}(\tau z)\right) \forall z \in\left[0, y_{0}\right], \\
& L_{-}(z)=\frac{1}{\tau} L_{+}\left(L_{+}(\tau z)\right) \forall z \in\left[y_{0}, 1\right] .
\end{aligned}
$$

The functions $L_{+}$and $L_{-}$are respectively holomorphic on complex neighborhoods of $\left[0, y_{0}\right)$ and $\left(y_{0}, 1\right]$ and they are $\mathscr{C}^{r}$ at $y_{0}$. They satisfy:

$$
L_{+}(0)=1, L_{ \pm}\left(y_{0}\right)=0, L_{+}\left(\tau y_{0}\right)=y_{0}, \tau L_{+}^{\prime}\left(\tau y_{0}\right)=-\lambda .
$$

Their inverse functions are respectively denoted $S_{+}$and $S_{-}$,

$$
S_{ \pm}(\zeta)=U\left( \pm \zeta^{1 / r}\right)
$$

\footnotetext{
${ }^{1}$ A complex function $h$ is called a Herglotz, or Herglotzian function if it is holomorphic in $\mathbf{C}_{+}$ $\cup \mathbf{C}_{-}, h\left(z^{*}\right)=h(z)^{*}$, and $h\left(\mathbf{C}_{+}\right) \subset \overline{\mathbf{C}}_{+}$; then $-h$ is an anti-Herglotz or anti-Herglotzian function
} 
$S_{+}$is holomorphic and anti-Herglotzian in $\mathbf{C}_{+} \cup \mathbf{C}_{-} \cup\left(0, \tau^{-2}\right)$. It is continuous and strictly decreasing on $\left[0, \tau^{-2}\right] . S_{-}$is holomorphic and Herglotzian in $\mathbf{C}_{+} \cup \mathbf{C}_{-}$ $\cup\left(0, \tau^{-1}\right)$, continuous and strictly increasing on $\left[0, \tau^{-1}\right]$. The domains of analyticity of $L_{ \pm}$are just the images given by $S_{ \pm}$of their respective cut-planes of analyticity. The identity

$$
L_{+}(\tau U(z))=U(-\lambda z)
$$

holds for all $z \in \Omega(\lambda)$.

\section{The Tangent Map to the Doubling Operator}

Here we adopt the point of view that, given $g, f$, etc. satisfying all the assumptions of Sect. 2 for some fixed $r$, with the corresponding values of $\lambda, \tau, y_{0}$ etc., we can e.g. regard $f$ as a fixed point of a doubling operator $\mathscr{R}$, defined by

$$
\mathscr{R}: F \mapsto-\frac{1}{\lambda} F \circ q \circ F \circ \tau .
$$

Here, $\lambda$ and $\tau$ have values independent of $F$. Note that rewriting the doubling operator in terms of $f$ (or $L$, or $U$ ) is merely a change of coordinates in function space. On the contrary maintaining $\lambda$ fixed [instead of taking it to be $-F(1)$ ] means a significant alteration of the "correct" doubling operator. This causes wellknown troubles (see e.g. [CEL], where the cure is also indicated) but it simplifies the task at hand in this paper. If we formally write $F=f+\delta f$, with $\delta f$ an infinitesimal variation of $f$, the corresponding variation $\delta \mathscr{R}_{f} F$ is given by:

$$
\delta \mathscr{R}_{f} F(z)=-\frac{1}{\lambda} \delta f\left(L_{+}(\tau z)\right)-\frac{r}{\lambda} f^{\prime}\left(L_{+}(\tau z)\right) f(\tau z)^{r-1} \delta f(\tau z) \equiv\left(D \mathscr{R}_{f} \delta f\right)(z) .
$$

It is the spectrum of the linear operator $D \mathscr{R}_{f}$ which we study in this paper.

Our bounds will crucially depend on the more convenient representation in terms of $\delta f / f^{\prime}$. Dividing both sides of (3.2) by

$$
f^{\prime}(z)=-\frac{r \tau}{\lambda} f^{\prime}\left(L_{+}(\tau z)\right) f(\tau z)^{r-1} f^{\prime}(\tau z),
$$

we see that, considered as acting on $\delta f / f^{\prime}$, the linear operator of (3.2) takes the form:

$$
(T v)(z)=\frac{v\left(L_{+}(\tau z)\right)}{\tau L_{+}^{\prime}(\tau z)}+\frac{v(\tau z)}{\tau} .
$$

This relatively simple form of (3.4) is easily seen from the identities

$$
\frac{\delta f}{f^{\prime}}=\frac{\delta f}{f} \frac{f}{f^{\prime}}=\frac{\delta L}{r L}, \quad \frac{r L}{L^{\prime}}=\frac{\delta L}{L^{\prime}}, \frac{\delta f}{f^{\prime}} \circ U=-\delta U, \text { etc. }
$$

Let us spell out in more detail the relationship between the operator $T$ as defined in (3.4) and the operator of (3.2), which, in less heuristic notation, can be 
written as

$$
(D \mathscr{R} \varphi)(z)=-\frac{1}{\lambda} \varphi\left(L_{+}(\tau z)\right)-\frac{r}{\lambda} f^{\prime}\left(L_{+}(\tau z)\right) f(\tau z)^{r-1} \varphi(\tau z)
$$

If we define the linear operator $Q$ by

$$
(Q \varphi)(z)=\frac{\varphi(z)}{f^{\prime}(z)}
$$

then

$$
Q D \mathscr{R}=T Q, T=Q D \mathscr{R} Q^{-1}
$$

The domains of these operators will be chosen to be certain spaces of functions holomorphic in complex neighborhoods of $[0,1]$ in such a way that these equations make sense. The spectrum of $D \mathscr{R}$ will then be the same as that of $T$.

It is natural to expect (and true) that $T$ will preserve the class of functions holomorphic in the same domain as $f$. Indeed recall that $U$ is holomorphic in $\Omega(\lambda)$ which it maps bijectively onto a certain bounded open subset $\Delta$ of $\mathbf{C}$, on which $f$ is holomorphic. By (2.7), $\tau \Delta \subset \Delta$. The identity (2.15) shows that $L_{+} \circ \tau$ is analytic on $\Delta$ and maps it into itself. But $L(\tau \Delta)=U(-\lambda \Omega(\lambda))$ is not relatively compact in $\Delta$ since their non-real points are the same. We will therefore use a sub-domain of $\Delta$ to make $T$ analyticity-improving. A convenient choice is given by

$$
\Delta_{1}=U\left(\left\{z:|z|<\frac{1}{\lambda}\right\}\right), \quad \Delta_{0}=U(\{z:|z|<u(-1)\}) .
$$

Note that $\Delta_{0} \Subset \Delta_{1}$ since

$$
1=u(-\lambda)<u(-1)<u(-1 / \lambda)=x_{0} / \lambda .
$$

Lemma 3.1. If $v$ is a real function on $[0,1]$ which extends to a holomorphic function on $\Delta_{0}$, then Tv extends to a holomorphic function on $\Delta_{1}$. For every $\Delta^{\prime} \Subset \Delta_{1}$ one has

$$
\sup _{z \in \Delta^{\prime}}|(T v)(z)| \leqq \frac{1}{\tau} \sup _{z \in \Delta^{\prime}}\left(1+\left|\frac{1}{L_{+}^{\prime}(\tau z)}\right|\right) \cdot \sup _{y \in \Delta_{0}}|v(y)| .
$$

Proof. We shall use the following simple fact: if a Herglotz or anti-Herglotz function is holomorphic on a real segment $(a, b)$ and maps it into the real segment $\left(a^{\prime}, b^{\prime}\right)$, then it maps the disk with diameter $(a, b)$ into the disk with diameter $\left(a^{\prime}, b^{\prime}\right)$. (See e.g. [E2].)

We claim now that $\tau \Delta_{1} \subset \Delta_{0}$ and $L_{+}\left(\tau \Delta_{1}\right) \subset \Delta_{0}$. Indeed, let $z=U(\zeta)$, for some $|\zeta|<1 / \lambda$. Then $\tau z=U(u(-\lambda \zeta))$, and $u(-\lambda \zeta)$ is contained in the disk with diameter $(0, u(-1))$; hence $\tau z \in \Delta_{0}$. On the other hand, by $(2.15)$, we have $L_{+}(\tau z)=U(-\lambda \zeta)$ and this is also in $\Delta_{0}$. The derivative of $L_{+} \circ \tau$ tends to zero near $y_{0} / \tau$. But its reciprocal is bounded in modulus in any $\Delta^{\prime} \Subset \Delta_{1}$. This completes the proof of the lemma.

We denote by $\mathscr{B}$ the Banach space of holomorphic, bounded functions on $\Delta_{0}$ which are real on $[0,1]$, equipped with the "sup" norm. It follows from Lemma 3.1 that $T \mathscr{B} \subset \mathscr{B}$ and $T$ is a compact linear operator on $\mathscr{B}$ whose eigenvalues form an exponentially decaying sequence. 
We use the following lemma to take advantage of the simple form of $T$ :

Lemma 3.2. The function $L_{+}$is convex on $\left[0, y_{0}\right]$, and the function $L_{-}$is convex on $\left[y_{0}, 1\right]$.

Proof. By our general assumptions, the function $U$ is holomorphic and antiHerglotzian in the cut plane $\Omega(\lambda)$, described by (2.3). As such it has positive Schwarzian derivative on the interval $\left(-\lambda^{-1}, \lambda^{-2}\right)$, i.e. $\phi=U^{\prime \prime} / U^{\prime}$ satisfies $2 \phi^{\prime} / \phi^{2}$ $-1 \geqq 0$. Integrating this inequality gives

$$
-\frac{2 \lambda}{1+\lambda z} \leqq \frac{U^{\prime \prime}(z)}{U^{\prime}(z)} \leqq \frac{2 \lambda^{2}}{1-\lambda^{2} z} .
$$

By (2.14),

$$
-\frac{S_{ \pm}^{\prime \prime}(\zeta)}{S_{ \pm}^{\prime}(\zeta)}=\frac{1}{r \zeta}\left[r-1-z \frac{U^{\prime \prime}(z)}{U^{\prime}(z)}\right] \text { with } \quad z= \pm \zeta^{1 / r} .
$$

We now use the lower bound for $r$ obtained in [E1]:

$$
r>\frac{1+\lambda^{2}}{1-\lambda^{2}} \text {. }
$$

For $z=\zeta^{1 / r}>0$ we find:

$$
-\frac{S_{+}^{\prime \prime}(\zeta)}{S_{+}^{\prime}(\zeta)}>\frac{1}{r \zeta}\left[\frac{1+\lambda^{2}}{1-\lambda^{2}}-\frac{1+\lambda^{2} z}{1-\lambda^{2} z}\right] .
$$

This is positive for $z<1$. For $z=-\zeta^{1 / r} \leqq 0$, we get:

$$
-\frac{S_{-}^{\prime \prime}(\zeta)}{S_{-}^{\prime}(\zeta)}>\frac{1}{r \zeta}\left[\frac{1+\lambda^{2}}{1-\lambda^{2}}-\frac{1-\lambda z}{1+\lambda z}\right] .
$$

This is positive for $-\lambda \leqq z \leqq 0$. Thus:

$$
-\frac{S_{+}^{\prime \prime}(\zeta)}{S_{+}^{\prime}(\zeta)}>0 \forall \zeta \in[0,1], \quad-\frac{S_{-}^{\prime \prime}(\zeta)}{S_{-}^{\prime}(\zeta)}>0 \quad \forall \zeta \in[0, \tau] .
$$

To see that the inequalities (3.15) remain strict even in the limit $r \rightarrow \infty$, we rewrite $r$ in $(3.11)$ as $\log (1 / \tau) / \log (1 / \lambda)$ and, using again the bounds $(3.10)$, and $\log (1 / \lambda)$ $<1 / \lambda-1$, we find:

$$
-\frac{S_{+}^{\prime \prime}(\zeta)}{S_{+}^{\prime}(\zeta)}>\frac{1}{\zeta}\left[1-\frac{1+\lambda^{2}}{\lambda(1+\lambda) \log (1 / \tau)}\right] \forall \zeta \in(0,1],
$$

and exactly the same inequality for $S_{-}^{\prime \prime} / S_{-}^{\prime}$ on $(0, \tau]$. This proves that $L_{+}$and $L_{-}$are convex on $\left[0, y_{0}\right]$ and $\left[y_{0}, 1\right]$ respectively. This completes the proof of Lemma 3.2.

Corollary 3.3. For all $z \in[0,1]$, we have $L_{+}(\tau z)>\tau z$ and $L_{+}^{\prime}(\tau z)<-1$.

Proof. By the monotonicity and convexity of $L_{+}$it suffices to prove this for $z=1$. Applying the functional equation (2.11) and its derivative at $z=0$ gives

$$
L(1)=-\tau, \quad L^{\prime}(1)=-1 .
$$


Reapplying them at $z=1$ gives

$$
L(L(\tau))=\tau^{2}, \quad L^{\prime}(L(\tau)) L^{\prime}(\tau)=1 .
$$

It follows that $L(\tau)<y_{0}$, and also $L(\tau)>\tau$. Otherwise $L(L(\tau) / \tau)$ would be in $[-1,1]$, contradicting

$$
L(L(\tau) / \tau)=-L\left(\tau^{2}\right) / \tau<-L\left(\tau y_{0}\right) / \tau=-y_{0} / \tau<-1 .
$$

The convexity of $L_{+}$implies $-L^{\prime}(\tau)>-L^{\prime}(L(\tau))$ and hence $-L^{\prime}(\tau)>1$ by (3.18).

From the convexity of $L_{ \pm}$we can now derive, following an idea of [CE], the existence of invariant cones for the operator $T$. However, the cones we define here do not coincide with the cones defined there because of the use of $v=\delta f / f^{\prime}$ instead of $\delta g$. (The cones of [CE] could not be shown to be invariant under the tangent map for $r$ much above 2 because of the lack of concavity of $g$ on $\left(x_{0}, 1\right]$.)

Definition. Define $\Gamma_{1}$ as the set of real $\mathscr{C}^{1}$ functions $v$ on $[0,1]$ for which

i) $v(z) \geqq 0$ for all $z \in[0,1]$,

ii) $v^{\prime}(z) \leqq 0$ for all $z \in[0,1]$.

We also define $\Gamma=\Gamma_{1} \cap \mathscr{B} . \Gamma$ is a closed cone with non-empty interior in $\mathscr{B}$.

Lemma 3.4. The tangent map $T$ maps $\Gamma_{1}$ into itself. Furthermore, $T^{2}$ maps any nonzero vector in $\Gamma$ into the interior of $\Gamma$.

Proof. Suppose $v \in \Gamma_{1}$. Then, since (by Corollary 3.3) for any $z \in[0,1], L_{+}(\tau z)>\tau z$, and since $v$ is decreasing,

$$
\tau(T v)(z) \geqq v(\tau z)\left[1+1 / L_{+}^{\prime}(\tau z)\right] .
$$

This is non-negative since $L_{+}^{\prime}(\tau z)<-1$ by Corollary 3.3. Furthermore

$$
(T v)^{\prime}(z)=v^{\prime}\left(L_{+}(\tau z)\right)-\frac{v\left(L_{+}(\tau z)\right) L_{+}^{\prime \prime}(\tau z)}{L_{+}^{\prime}(\tau z)^{2}}+v^{\prime}(\tau z) .
$$

The point is now that all three terms of this formula are non-positive, so that $T v$ is indeed in $\Gamma_{1}$. The interior of $\Gamma$ is clearly composed of those $v$ for which the inequalities defining $\Gamma$ are all strict. Suppose $v \in \Gamma$ is not 0 . If $v(z)$ vanished for some $z \in[0,1)$, it would have to vanish on $[z, 1]$, hence everywhere by analyticity, i.e. 1 is the only place in $[0,1]$ where $v$ can vanish. But $T v$ cannot vanish even at 1 by (3.20). Furthermore the middle term in (3.21) cannot vanish in $(0,1]$, and can vanish at 0 only if $v(1)=0$. Hence $T^{2} v$ is in the interior of $\Gamma$ as claimed.

\section{Inequalities and Numerical Bounds}

Suppose $v_{e} \in \Gamma \backslash\{0\}$ and $T v_{e}=\varrho v_{e}$. Then $v_{e}$ is in the interior of $\Gamma$ by Lemma 3.4, and

$$
\varrho v_{e}(0)=\frac{v_{e}(1)}{\tau L^{\prime}(0)}+\frac{v_{e}(0)}{\tau}>v_{e}(0)\left[\frac{1}{\tau}+\frac{1}{\tau L^{\prime}(0)}\right]>v_{e}(0)\left(\frac{1}{\tau}-\frac{1}{\lambda}\right) .
$$

The last inequality uses $-\tau L^{\prime}(0)>\lambda$ due to the convexity of $L_{+}$. The middle inequality is strict because $v_{e}$ is in the interior of $\Gamma$, so that $v_{e}(1)<v_{e}(0)$. Finally, 
since $v_{e}(1)>0$, we get the inequality announced in the Introduction:

$$
\frac{1}{\tau}-\frac{1}{\lambda}<\frac{1}{\tau}+\frac{1}{\tau L^{\prime}(0)}<\varrho<\frac{1}{\tau} .
$$

Applying the theorem of Krein and Rutman [KR] we obtain from Lemma 3.4:

Lemma 4.1. As an operator on $\mathscr{B}, T$ possesses an eigenvalue of largest modulus $\delta$ which is real and positive. The spectral subspace corresponding to this eigenvalue is one-dimensional and generated by an element of the interior of $\Gamma$ which is (up to rescaling) the only eigenvector of $T$ in $\Gamma$. This eigenvalue satisfies the bounds (4.2). The adjoint $T^{*}$ of $T$ has a unique eigenvector $\varphi_{e}$ in the cone $\Gamma^{*}$ dual to $\Gamma$ (i.e., the set of continuous linear functionals on $\mathscr{B}$ which take positive values on all elements of $\Gamma$ ) and the corresponding eigenvalue is $\delta$.

At $r=\infty$, we can use the rigorous numerical bounds obtained in [EW1], written here just as ordinary numbers, not as intervals:

$$
y_{0}=0.391132999351022542, \tau=0.033381055, L_{+}^{\prime}(0)=-67.42069 \text {. }
$$

This gives

$$
\frac{1}{\tau}=29.957112, \frac{1}{\tau}-1=28.957112, \frac{1}{\tau}\left(1+\frac{1}{L^{\prime}(0)}\right)=29.5128,
$$

to be compared with the following numerical estimate of $\delta$ :

$$
\delta=29.5763 \text {. }
$$

This shows that the bounds (4.2) become rather satisfactory at $r=\infty$. They are poorer at, e.g. $r=2$, where

$$
\begin{gathered}
\delta=4.669201609, \quad \text { while } \frac{1}{\tau}=6.26454783121704, \frac{1}{\tau}-\frac{1}{\lambda}=3.7616, \\
f^{\prime}(0)=-1.52763299703630145, \frac{1}{\tau}\left(1+\frac{1}{L^{\prime}(0)}\right)=4.2141 .
\end{gathered}
$$

Acknowledgements. This work was begun during the first author's stay at the IHES, where he profited from the usual warm hospitality. Further support was received from the Fonds National Suisse.

\section{References}

[CEL] Collet, P., Eckmann, J.-P., Lanford, O.E., III: Universal properties of maps on the interval. Commun. Math. Phys. 76, 211-254 (1980)

[CE] Campanino, M., Epstein, H.: On the existence of Feigenbaum's fixed point. Commun. Math. Phys. 79, 261-302 (1981)

[CT] Coullet, P., Tresser, C.: Itération d'endomorphismes et groupe de renormalisation. J. Phys. Colloque C539, C5-25 (1978). CRAS Paris 287 A (1978)

[E1] Epstein, H.: New proofs of the existence of the Feigenbaum functions. Commun. Math. Phys. 106, 395-426 (1986)

[E2] Epstein, H.: Fixed points of composition operators. In: Non-linear evolution and chaotic phenomena. Gallavotti, G., Zweifel, P. (eds.). Plenum Press: New York 1988 
[E3] Epstein, H.: Fixed points of composition operators. II. Nonlinearity 2, 305-310 (1989)

[EW1] Eckmann, J.-P., Wittwer, P.: Computer methods and Borel summability applied to Feigenbaum's equation. Lecture Notes in Physics, vol. 227. Berlin, Heidelberg, New York: Springer 1985

[EW 2] Eckmann, J.-P., Wittwer, P.: A complete proof of the Feigenbaum conjectures. J. Stat. Phys. 46, 455-477 (1987)

[F] Feigenbaum, M.J.: Quantitative universality for a class of non-linear transformations. J. Stat. Phys. 19, 25-52 (1978). Universal metric properties of non-linear transformations. J. Stat. Phys. 21, 669-706 (1979)

[KR] Krein, M.G., Rutman, M.A.: Usp. Mat. Nauk 3, 1, 3-95 (1948); English Translation: Functional analysis and measure theory. Providence: Am. Math. Soc. 1962

Communicated by A. Jaffe

Received June 20, 1989

Note added in proof. Using the upper bounds on $\tau$ given in [E1], it is easy to see that $(1 / \tau-1 / \lambda)>1$ (and hence $\delta>1$ ) for all $r>1$. 
